\title{
A preliminary evaluation of VEGF-A, VEGFR1 and VEGFR2 in patients with well-controlled type 2 diabetes mellitus*
}

\author{
Barbara RUSZKOWSKA-CIASTEK ${ }^{\dagger 1}$, Alina SOKUP ${ }^{1}$, Maciej W. SOCHA ${ }^{2}$, Zofia RUPRECHT ${ }^{3}$, \\ Lidia HAŁAS ${ }^{1}$, Barbara GÓRALCZYK ${ }^{1}$, Krzysztof GÓRALCZYK ${ }^{1}$, Grażyna GADOMSKA ${ }^{1}$, Danuta ROŚĆ ${ }^{1}$ \\ ('Department of Pathophysiology, Collegium Medicum in Bydgoszcz, Nicolaus Copernicus University in Toruń, Poland) \\ ( ${ }^{2}$ Department of Obstetrics, Gynecology and Oncological Gynecology, Collegium Medicum in Bydgoszcz, \\ Nicolaus Copernicus University in Toruń, Poland) \\ ( ${ }^{3}$ Department of Endocrinology and Diabetology, Collegium Medicum in Bydgoszcz, Nicolaus Copernicus University in Torun, Poland) \\ †E-mail: ruszkowska.basia@gmail.com \\ Received Jan. 25, 2014; Revision accepted Apr. 17, 2014; Crosschecked May 12, 2014
}

\begin{abstract}
Objective: Decompensated chronic hyperglycemia often leads to late microvascular complications such as retinopathy, diabetic foot syndrome, and diabetic kidney disease. The aim of this study was to determine the concentration of vascular endothelial growth factor A (VEGF-A) and its receptors in patients with well-controlled diabetes. Methods: The study was conducted on 31 patients with well-controlled type 2 diabetes without micro- or macroangiopathy. Thirty healthy volunteers were enrolled in a control group. Serum concentrations of VEGF-A, VEGF receptors 1 and 2 (VEGFR1 and VEGFR2), fasting glucose, and lipid profiles were measured, and the plasma concentration of glycated hemoglobin (HbA1c) was determined. Results: No significant differences were observed between the concentration of VEGF-A, VEGFR1 or VEGFR2 in the subject group and that in the control group. Positive correlations were noted between the levels of VEGF-A, VEGFR2, and triglyceride, and there was a negative correlation between the levels of VEGFR2 and high-density lipoprotein (HDL)-cholesterol in the study group. Conclusions: The concentrations of VEGF-A and its receptors 1 and 2 in patients with well-controlled diabetes are comparable to those of healthy individuals, which may indicate that appropriate control of glucose levels delays the occurrence of vascular complications. A negative correlation between VEGFR2 and HDL-cholesterol levels, and positive correlations between VEGF-A, VEGFR2, and triglyceride levels, suggest that lipid abnormalities occurring in diabetes may be involved in the modulation of angiogenesis.
\end{abstract}

Key words: Type 2 Diabetes, Angiogenesis, Lipid abnormalities, Glycated hemoglobin (HbA1c) doi:10.1631/jzus.B1400024 Document code: A $\quad$ CLC number: R587.1

\section{Introduction}

Type 2 diabetes mellitus, along with cardiovascular diseases, cancers, and chronic respiratory diseases, is classified as a non-communicable disease (NCD) and is a major cause of human morbidity and mortality worldwide (World Health Organization, 2011). In 2012, diabetes caused 4.8 million deaths in the world and there were 371 million diabetic patients (International Diabetes Federation, 2012; Olokoba et

\footnotetext{
" Project supported by the Collegium Medicum in Bydgoszcz, Nicolaus Copernicus University in Toruń, Poland

(C) Zhejiang University and Springer-Verlag Berlin Heidelberg 2014
}

al., 2012). By 2030, morbidity is expected to increase to 522 million, of whom 439 million will suffer from type 2 diabetes (Olokoba et al., 2012).

The main problem is still late, often random, clinical diagnosis of type 2 diabetes. Latent and oligosymptomatic onset results in vascular complications in more than $25 \%$ of patients at diagnosis (Olokoba $e t$ al., 2012). This relates to damage to small arterioles (microangiopathy) and large vessels (macroangiopathy) and hemostatic disorders (diabetic thrombophilia), which in turn lead to multiple organ dysfunction.

The basis of the development of late diabetic complications is endothelial dysfunction, which leads to impaired function of many processes such as blood 
coagulation, fibrinolysis, and the severity of the inflammatory response (Basha et al., 2012). Also noted is an incorrect expression of multiple pro-angiogenic factors, which is manifested by dysregulation of the angiogenesis process and underlies vascular complications in diabetes (Jansson, 2007).

In the angiogenesis process, the most potent mitogens acting on endothelial cells (ECs) are the vascular endothelial growth factor (VEGF) and basic fibroblast growth factor (bFGF). The expression of VEGF, which occurs under the influence of hypoxia inducible factor-1 (HIF-1), starts and maintains a neovascularization process (Zielonka, 2004; Skóra et al., 2006). The stimulation of a type 2 receptor (VEGFR-2) specific for VEGF (fetal liver kinase-1 (Flk-1) or kinase domain region (KDR)) with tyrosine kinase activity by activating the phosphoinositol-3kinase/protein kinase B (PI3K/Akt) pathway activates endothelial nitric oxide synthase (eNOS). This enhances the release of nitric oxide (NO) which extends and increases the permeability of the vessel, which is vital for the start of angiogenesis. VEGF also acts through the receptor VEGFR1 (Fms-like tyrosine kinase-1 (Flt-1)), which, in response, generates vascular sprouting (Barańska et al., 2005; Stuttfeld and Ballmer-Hofer, 2009).

Processes occurring in diabetes such as hyperglycemia, insulin resistance, hypertension, dyslipidemia, central obesity, and impaired NO synthesis have an effect on blood flow in the vessels and cause tissue hypoxia. Hypoxia is a signal for the induction of angiogenesis and the expression of many genes, including VEGF and VEGFR2, which, due to their functions, may have an impact on the development of diabetic complications (Jansson, 2007).

A review of the literature suggests that the expression of VEGF and its receptor 2 increases significantly during the course of pathological conditions such as diabetic retinopathy and nephropathy (Kubisz et al., 2010; Mahdy et al., 2010). However, there are no unambiguous data on the role of VEGF-A, VEGFR1, or VEGFR2 in the pathogenesis of wellcontrolled diabetes. With that in mind, the aim of this study was to determine the concentrations of VEGF-A and its receptors 1 and 2 in patients with well-controlled type 2 diabetes mellitus without micro- or macroangiopathy.

\section{Materials and methods}

\subsection{Subjects}

The study included 31 patients diagnosed with type 2 diabetes mellitus under the care of the Endocrinology and Diabetology Clinic of Dr. A. Jurasz University Hospital, Poland. The study group consisted of 13 females aged 49-74 years (mean 63 years) and 18 males aged $46-79$ years (mean 64 years). To qualify for the study, patients had to satisfy the following criteria: a level of glycated hemoglobin ( $\mathrm{HbAlc}$ ) of $\leq 6.5 \%$ and no vascular complications, assessed by determining the concentration of albumin in the urine (to exclude diabetic kidney disease), an eye examination (to exclude the presence of diabetic retinopathy), as well as no symptoms of ischaemic heart disease, no symptoms of lower limb ischemia (measured by testing the pressure of the arteries of the lower limbs to calculate the ankle-brachial index: selected patients had an index of 0.9-1.15), and no diabetic foot syndrome. The control group consisted of 30 healthy individuals: 19 females aged $45-57$ years (mean 51 years) and 11 males aged 40-67 years (mean 50 years).

A medical history interview conducted by a medical specialist provided information about the general condition of the patients and the presence of co-existing diseases such as hypertension, obesity, and hyperlipoproteinaemia. The criterion for the diagnosis of obesity was a body mass index (BMI) of over $30 \mathrm{~kg} / \mathrm{m}^{2}$, and the criteria for high blood pressure were systolic blood pressure $\geq 140 \mathrm{mmHg}$ and diastolic blood pressure $\geq 90 \mathrm{mmHg}$ (Table 1). The mean duration of type 2 diabetes mellitus in patients was 8.1 years. Among all the patients, 26 had used antiplatelet drugs and 2 had used anticoagulant therapy. None of the patients received fibrinolytic drugs. All the patients received antihyperglycemic treatment: insulin, metformin or sulfonylurea derivatives, depending on the indication. The patients also had received statins and ACE inhibitor drugs.

The patients from all the groups were informed about the aim of the study and its course. They expressed informed consent for participation in the study and the research was approved by the Bioethics Committee of the Collegium Medicum in Bydgoszcz, Nicolaus Copernicus University in Torun, Poland (No. KB 89/2009). 
Table 1 Selected clinical data of patients with type 2 diabetes in relation to gender

\begin{tabular}{|c|c|c|c|c|c|c|}
\hline \multirow{2}{*}{ Group } & \multirow{2}{*}{$n$} & \multirow{2}{*}{$\begin{array}{l}\text { Mean age } \\
\text { (year) }\end{array}$} & \multicolumn{2}{|c|}{ Number of patients } & \multirow{2}{*}{$\begin{array}{l}\text { Triglycerides } \\
(\mathrm{mg} / \mathrm{dl})\end{array}$} & \multirow{2}{*}{$\begin{array}{l}\text { LDL-cholesterol } \\
(\mathrm{mg} / \mathrm{dl})\end{array}$} \\
\hline & & & Hypertension & Hyperlipoproteinemia & & \\
\hline \multicolumn{7}{|l|}{ Study } \\
\hline Female & 13 & 63 & 12 & 8 & 138 & 115 \\
\hline Male & 18 & 64 & 13 & 11 & 131 & 118 \\
\hline Control & 30 & 51 & & & 108 & 104 \\
\hline Group & \multicolumn{2}{|c|}{$\begin{array}{c}\text { HDL-cholesterol } \\
(\mathrm{mg} / \mathrm{dl})\end{array}$} & $\begin{array}{c}\text { Cholesterol } \\
(\mathrm{mg} / \mathrm{dl})\end{array}$ & \multicolumn{2}{|c|}{$\begin{array}{c}\text { BMI }\left(\mathrm{kg} / \mathrm{m}^{2}\right) / \\
\text { number of patients }\end{array}$} & $\begin{array}{l}\text { HbAlc (mmol/mol)/ } \\
\text { GSP HbA1c (\%) }\end{array}$ \\
\hline \multicolumn{7}{|l|}{ Study } \\
\hline Female & \multicolumn{2}{|c|}{48} & 185 & \multicolumn{2}{|c|}{$35.85 / 8$} & $48 / 6.5$ \\
\hline Male & \multicolumn{2}{|c|}{45} & 191 & \multicolumn{2}{|c|}{$34.00 / 6$} & $48 / 6.5$ \\
\hline Control & \multicolumn{2}{|c|}{57} & 186 & \multicolumn{2}{|c|}{23} & $<26 /<4.5$ \\
\hline
\end{tabular}

LDL-cholesterol: low-density lipoprotein-cholesterol; HDL-cholesterol: high-density lipoprotein-cholesterol; BMI: body mass index; IFCC: International Federation of Clinical Chemistry; NGSP: National Glycohemoglobin Standardization Program

\subsection{Measurements}

The materials used for the studies consisted of venous blood collected without stasis from an elbow vein. The blood was collected between 7:30 and 9:30 am after half an hour of rest in a fasting state. To determine angiogenic factors, $4.5 \mathrm{ml}$ of blood was collected into tubes without anticoagulant. The sample was centrifuged for $20 \mathrm{~min}$ at $4{ }^{\circ} \mathrm{C}$ at $3000 \times g$ and subjected to further analytical procedures. In the serum from the study and control groups, the concentrations of VEGF-A, VEGFR1, VEGFR2, the lipid profile, and fasting glucose were determined. In addition, $4.5 \mathrm{ml}$ of blood was collected into tubes containing sodium versene (ethylenediaminetetraacetic acid (EDTA)) to determine the level of HbAlc; the plasma received from the study group was directly subjected to further analytical procedures.

The VEGF-A concentration was determined using the Quantikine VEGF immunoassay, the VEGFR1 concentration using the Quantikine Human sVEGFR1/ Flt-1 immunoassay, and the VEGFR2 concentration using the Quantikine Human sVEGFR2/KDR/Flk-1 immunoassay. All test kits were supplied by R\&D Systems, Inc. The method was based on the reaction enzyme immunoassay (ELISA). The parameters of the lipid profile, fasting glucose, and $\mathrm{HbAlc}$ concentrations were determined by specific tests using an Abbott Clinical Chemistry Analyzer Architect c8000.

\subsection{Statistical analysis}

The statistical analysis was performed using Statistica 10.0 software (StatStoft ${ }^{\circledR}$, Cracow, Poland).
The Shapiro-Wilk test was used to assess the normality of data distributions. Parameters with values deviating from a normal distribution were described by the median $(\mathrm{Me})$ and lower $(\mathrm{Q} 1)$ and upper $(\mathrm{Q} 3)$ quartiles. The variables that were close to a normal distribution were expressed as arithmetic means and standard deviations (SDs). The differences between the parameters in each group were assessed using the non-parametric U-Mann-Whitney rank-sum test for variables with a non-normal distribution, or by Student's $t$-test for normally distributed data. To assess the correlation between the parameters, the Spearman $(R)$ coefficient was used. $P$ values $<0.05$ were considered significant.

\section{Results}

Table 2 shows the concentrations of VEGF-A, VEGFR1, and VEGFR2 in the study group compared with those in the control. There were no significant differences in the parameters between the two groups.

Table 2 Concentrations of VEGF-A, VEGFR1, and VEGFR2 in the study group compared with those in the control group

\begin{tabular}{cccc}
\hline Group & $\begin{array}{c}\text { VEGF-A } \\
(\mathrm{pg} / \mathrm{ml})\end{array}$ & $\begin{array}{c}\text { VEGFR1 } \\
(\mathrm{pg} / \mathrm{ml})\end{array}$ & $\begin{array}{c}\text { VEGFR2 } \\
(\mathrm{pg} / \mathrm{ml})\end{array}$ \\
\hline Study & 11.15 & 144.33 & 12794.22 \\
$(n=31)$ & $(7.22 ; 17.06)$ & $(89.32 ; 226.84)$ & $(2411.12)$ \\
Control & 12.13 & 158.08 & 13625.84 \\
$(n=30)$ & $(9.18 ; 16.07)$ & $(89.32 ; 240.59)$ & $(2397.41)$ \\
\hline$P$-value & 0.24 & 0.73 & 0.24 \\
\hline
\end{tabular}

Data are expressed as Me (Q1; Q3) or mean (SD) 
Table 3 Spearman $(R)$ correlation coefficients of the parameters analyzed with the lipid profile and HbA1c in patients with type 2 diabetes

\begin{tabular}{|c|c|c|c|c|c|c|c|c|c|c|}
\hline \multirow{2}{*}{ Parameter } & \multicolumn{2}{|c|}{ Triglyceride } & \multicolumn{2}{|c|}{ Total cholesterol } & \multicolumn{2}{|c|}{ LDL-cholesterol } & \multicolumn{2}{|c|}{ HDL-cholesterol } & \multicolumn{2}{|c|}{$\mathrm{HbAlc}$} \\
\hline & $R$ & $P$ & $R$ & $P$ & $R$ & $P$ & $R$ & $P$ & $R$ & $P$ \\
\hline VEGF-A & $0.4899^{*}$ & 0.04 & 0.0136 & 0.96 & -0.1596 & 0.55 & 0.1775 & 0.51 & 0.3668 & 0.11 \\
\hline VEGFR1 & -0.2189 & 0.38 & -0.2217 & 0.36 & -0.1722 & 0.52 & -0.2388 & 0.36 & -0.3724 & 0.09 \\
\hline VEGFR2 & $0.5315^{*}$ & 0.03 & -0.1825 & 0.67 & -0.0500 & 0.93 & $-0.4957^{*}$ & 0.04 & -0.0870 & 0.78 \\
\hline
\end{tabular}

Table 3 shows the relationships between VEGF-A, its receptors and lipid parameters, as well as HbAlc. In the group of patients, there was a significant positive correlation between the concentrations of triglycerides and VEGF-A and VEGFR2. There was also a significant negative correlation between the highdensity lipoprotein (HDL)-cholesterol and VEGFR2 concentrations.

\section{Discussion}

The factor most widely known to be involved in angiogenesis, connected with chronic hyperglycemia and its consequences, is VEGF. VEGF stimulates the proliferation, differentiation, and survival of ECs. It is also an agent for endothelium-dependent vasodilation, increasing its permeability, and participates in cellular interstitial matrix remodeling (Kubisz et al., 2010). It is believed that this cytokine plays a key role in prenatal and postnatal angiogenesis, in both physiological (e.g., wound healing, pregnancy) and pathological states where excessive formation of blood vessels occurs, such as in tumors, rheumatoid arthritis, diabetic retinopathy, or diabetic nephropathy (Araszkiewicz et al., 2004). In the present study, no significant differences were observed between the concentration of VEGF-A in the group of patients with type 2 diabetes without vascular complications and that in the control group. This suggests that proper glycemic control considerably reduces the risk of vascular complications associated with elevated levels of VEGF, and poor glycemic control and its metabolic consequences can be considered as major factors in the increased expression of VEGF in diabetes (Swidzińska et al., 2006). This phenomenon is most probably due to polyol pathway abnormalities, increased non-enzymatic glycation of proteins, increased levels of angiotensin II, and hypoxia as a result of hyperglycemia (Cameron et al., 2001). A review of the literature indicates essential differences in the concentration of VEGF-A in patients with diabetes. Kubisz et al. (2010) recorded a significantly higher concentration of VEGF-A in the serum of patients with type 2 diabetes with normoalbuminuria, compared with a control group. However, no significant differences were noted between serum VEGF-A levels in patients with type 2 diabetes and accompanying microalbuminuria, compared with a group of healthy individuals. Kim et al. (2005) reported similar results for VEGF-A in patients with type 2 diabetes mellitus complicated by nephropathy, compared with a control group. However, different results were noted in the urine, where they found higher levels of VEGF-A in patients with type 2 diabetes complicated by nephropathy, compared with healthy people. Santilli et al. (2001) observed an increased concentration of serum VEGF in patients with type 1 diabetes mellitus complicated by retinopathy and diabetic nephropathy. Araszkiewicz et al. (2004) reported increased levels of VEGF-A in patients with type 1 diabetes, both in those with diabetic microangiopathy and in those without that disorder. Mahdy et al. (2010) conducted a study with a group of 55 patients with type 2 diabetes, including 10 patients without vascular complications, and reported a higher level of VEGF-A compared with a control group. However, sufficient glycemic control led to a reduction in the concentration of VEGF-A. Despite much evidence that abnormal glycemic control is conducive to the development of vascular complications, a definitive statement that the proper alignment of glucose completely eliminates that risk cannot be made. Increased VEGF production in diabetic patients may be the result of not only periodic states of hyperglycemia and hypoglycaemia, but also an effect of exogenous 
insulin administration or genetic factors (Kowalski et al., 2011). Studies suggest that fluctuations in the blood glucose levels cause the disorder known as cell pseudohypoxia that leads to the induction of VEGF synthesis (Araszkiewicz et al., 2004).

VEGFR2 is one of the three receptors for VEGF and is mainly responsible for its biological response. VEGF activation through VEGFR2 is the crucial mechanism stimulating the vascular endothelium and the concentration of VEGFR2 is strongly positively correlated with the amount of VEGF-A. No such dependence is observed in the resting endothelium (Gumbrecht, 2004). In the present study, there were no significant differences between the levels of VEGFR2 in the control group and those in the group of patients with well-controlled diabetes. Similar results were reported by Janota et al. (2003) who analyzed the expression of VEGF, angiopoietin 1, angiopoietin 2, and their receptors in the human placenta in healthy subjects and in patients with wellcontrolled type 1 diabetes. Patients with complications of diabetes, such as retinopathy or diabetic nephropathy, show a significant increase in the level of VEGFR2 compared with healthy individuals. Witmer et al. (2002) analyzed the expression of VEGFR2 in the retina of people with diabetic retinopathy and in the retina of mice with induced retinopathy. They found increased levels of VEGFR2 and VEGFR3, mainly leaking retinal microvessels, induced by the primary increase in VEGF (Witmer $e t$ al., 2002). Cooper et al. (1999) analyzed the changes in gene expression of VEGF and its receptors after streptozotocin-induced diabetes. VEGF and VEGFR2 measurements were taken 3 and 32 weeks after the administration of a pharmacological agent. The results showed an increase in the levels of mRNA for VEGF and VEGFR2 after 3 weeks of streptozotocin administration. However, no increase in long-term experimental diabetes was noted. The results confirmed the role of VEGF in mediating the changes occurring in the early stages of diabetes.

VEGFR1 (Flt-1) is found mainly in vascular ECs, monocytes, macrophages, trophoblasts of the placenta, and in kidney mesangial cells. A receptor is located on the hypoxia-dependent sequence (hypoxia responsive elements (HRE)) within the promoter of the gene. The stimulation of this receptor by the ligand is characterized by a poor response (Stuttfeld and
Ballmer-Hofer, 2009). Kim et al. (2005) studied 107 patients with type 2 diabetes with normoalbuminuria, microalbuminuria, and proteinuria, and recorded higher levels of VEGFR1 in the urine of patients with type 2 diabetes, compared with a control group. However, plasma levels of VEGF-A and VEGFR1 in the study group with normoalbuminuria, microalbuminuria, and proteinuria and the control group were similar.

An important risk factor for type 2 diabetes is obesity and strongly related disorders of lipid parameters. It is estimated that dyslipidemia occurs in $60 \%-80 \%$ of diabetic patients (Pacholczyk et al., 2008). The development, maturation, and plasticity of blood vessels are involved in the proper functioning of adipose tissue as a metabolic and endocrine organ. Angiogenesis is a key factor in adipogenesis (KiećWilk et al., 2006; Wada et al., 2011). The current study reports a positive correlation between serum triglyceride levels and VEGF, which is consistent with the study by Peczyńska et al. (2004), which reported patients with incipient diabetic angiopathy. Mahdy et al. (2010) also noted a positive correlation between lipid parameters (total cholesterol and low-density lipoprotein (LDL)-cholesterol) in patients with type 2 diabetes. In addition, Wada et al. (2011) also noted a positive correlation between serum triglyceride and VEGF-A levels. They showed that obesity and lipid disorders occur at the same time and influence the process of angiogenesis. In the present study, we observed a positive correlation between the levels of VEGFR2 and triglycerides and a negative correlation between the levels of VEGFR2 and HDL-cholesterol. In metabolic diseases, carbohydrate metabolism disorders often can be accompanied by abnormalities of the lipid profile. It is known that LDL-cholesterol is a factor which directly damages ECs. Fluctuations in blood glucose levels and anti-hyperglycemic treatment induce VEGF expression and consequently, an increase in VEGFR2 (Witmer et al., 2002). The negative correlation between the levels of VEGFR2 and HDL-cholesterol is an expression of the beneficial effects of HDL in lipid metabolism, which are impaired in diabetes. The absence of a correlation between VEGFR1 and lipid parameters may indicate that the stimulation of VEGF-A occurs primarily through VEGFR2 (Wada et al., 2011).

Hyperglycemia acts as toxin to the endothelium 
through increasing oxidative stress and inhibiting the bioavailability of $\mathrm{NO}$ and the formation of advanced glycation end products. Besides reducing the availability of NO, a high concentration of glucose in the blood increases the production of vasoconstrictor substances, especially endothelin-1 (Derzhko and Witkowska, 2006). Hyperglycemia-induced pathological mechanisms also affect the expression of VEGF and its type 1 and 2 receptors. The laboratory parameter used for evaluation of glucose control in the blood is the level of HbAlc. Kim et al. (2005) found no significant correlation between the concentrations of VEGF and VEGFR1 and the level of HbA1c. A similar result recorded in the present study might be due to the fact that one of the criteria for the qualifying patients was a level of $6.5 \% \mathrm{HbA} 1 \mathrm{c}$. The results indicate that well-controlled diabetes type 2 is a result of properly maintained glycemic control in such patients. This hypothesis was supported by the results of Mahdy et al. (2010) who measured the concentration of VEGF in patients with type 2 diabetes before glycemic control and after 4 months of monitoring, and observed a significant decrease in the concentration of VEGF in patients with glycemic control.

\section{Conclusions}

The concentrations of VEGF-A and its receptors 1 and 2 in patients with well-controlled diabetes were comparable to those in healthy individuals. This may suggest that properly controlled blood glucose levels reduce or delay the occurrence of vascular complications. The existence of a negative correlation between the levels of VEGFR2 and HDL-cholesterol and the positive correlations between the concentrations of VEGF-A, VEGFR2 and triglyceride, seems to suggest that lipid abnormalities occurring in diabetes may be involved in the modulation of angiogenesis.

\section{Compliance with ethics guidelines}

Barbara RUSZKOWSKA-CIASTEK, Alina SOKUP, Maciej W. SOCHA, Zofia RUPRECHT, Lidia HAŁAS, Barbara GÓRALCZYK, Krzysztof GÓRALCZYK, Grażyna GADOMSKA, and Danuta ROŚĆ declare that they have no conflict of interest.

All procedures followed were in accordance with the ethical standards of the responsible committee on human experimentation (institutional and national) and with the Hel- sinki Declaration of 1975, as revised in 2008 (5). Informed consent was obtained from all patients for being included in the study. Additional informed consent was obtained from all patients for which identifying information is included in this article.

\section{References}

Araszkiewicz, A., Zozulińska, D., Wierusz-Wysocka, B., 2004. Assessment of vascular-endothelial growth factor (VEGF) serum concentration in type 1 diabetic subject. Diabet. Dosw. Klin., 4(S3):197-201 (in Polish).

Barańska, P., Jerczyńska, H., Pawłowska, Z., 2005. Vascular endothelial growth factor-structure and functions. Postepy Biochem., 51(1):12-21 (in Polish).

Basha, B., Samuel, S.M., Triggle, C.R., et al., 2012. Endothelial dysfunction in diabetes mellitus: possible involvement of endoplasmic reticulum stress? Exp. Diabetes Res., 2012:481840. [doi:10.1155/2012/481840]

Cameron, N.E., Eaton, S.E.M., Cotter, M.A., 2001. Vascular factors and metabolic interactions in the pathogenesis of diabetic neuropathy. Diabetologia, 44(11):1973-1988. [doi:10.1007/s001250100001]

Cooper, M., Vranes, D., Youssef, S., et al., 1999. Increased renal expression of vascular endothelial growth factor (VEGF) and its receptor VEGFR-2 in experimental diabetes. Diabetes, 48(11):2229-2238. [doi:10.2337/diabetes. 48.11.2229]

Derzhko, R., Witkowska, M., 2006. Glucose metabolism disturbances and the risk of cardiovascular diseases. $A d v$. Clin. Exp. Med., 15(5):911-916.

Gumbrecht, J., 2004. Insulin and endothelium. Przew. Lek., 7:58-61 (in Polish).

International Diabetes Federation, 2012. Diabetes Atlas, 5th Ed. Brussels, Belgium.

Janota, J., Pomyje, J., Toth, D., et al., 2003. Expression of angiopoietic factors in normal and type-I diabetes human placenta: a pilot study. Eur. J. Obstet. Gynecol. Reprod. Biol., 111(2):153-156. [doi:10.1016/S0301-2115(03)00 204-5]

Jansson, P.A., 2007. Endothelial dysfunction in insulin resistance and type 2 diabetes. J. Intern. Med., 262(2): 173-183. [doi:10.1111/j.1365-2796.2007.01830.x]

Kieć-Wilk, B., Dudek, W., Dembińska-Kieć, A., 2006. Nutrigenomics, angiogenesis and obesity. Acta Angiol., 12(4):141-148.

Kim, H.N., Oh, H.J., Seo, A.J., et al., 2005. Vascular endothelial growth factor (VEGF) and soluble VEGF receptor FLT-1 in diabetic nephropathy. Kidney Int., 67(1):167177. [doi:10.1111/j.1523-1755.2005.00067.x]

Kowalski, J., Śliwczyńska-Rodziewicz, D., Kowalczyk, E., et al., 2011. Plasma nitric oxide and vascular endothelial growth factor levels in patients with metabolic syndrome and co-existing vascular complications. Pol. Merkur. Lekarski, 30(178):249-252 (in Polish).

Kubisz, P., Chudy, P., Staśko, J., et al., 2010. Circulating vascular endothelial growth factor in the normo- and/or 
microalbuminuric patients with type 2 diabetes mellitus. Acta Diabetol., 47(2):119-124. [doi:10.1007/s00592-0090127-2]

Mahdy, R.A., Nada, W.M., Hadhoud, K.M., et al., 2010. The role of vascular endothelial growth factor in the progression of diabetic vascular complications. Eye, 24(10): 1576-1584. [doi:10.1038/eye.2010.86]

Olokoba, A.B., Obateru, O.A., Olokoba, L.B., 2012. Type 2 diabetes mellitus: a review of current trends. Oman Med. $J ., 27(4): 269-273$. [doi:10.5001/omj.2012.68]

Pacholczyk, M., Ferenc, T., Kowalski, J., 2008. The metabolic syndrome. Part II: its mechanisms of development and its complications. Postepy Hig. Med. Dosw., 62:543-558 (in Polish).

Peczyńska, J., Urban, M., Urban, B., et al., 2004. Assessment of growth factor levels in adolescents with type 1 diabetes mellitus and the beginning of diabetic microangiopathy. Endokrynol. Diabetol. Chor. Przemiany Materii Wieku Rozw., 10(1):41-48 (in Polish).

Santilli, F., Spagnoli, A., Mohn, A., 2001. Increased vascular endothelial growth factor serum concentrations may help to identify patients with onset of type 1 diabetes during childhood at risk for developing persistent microalbuminuria. J. Clin. Endocrinol. Metab., 86(8):3871. [doi:10. 1210/jcem.86.8.7752]
Skóra, J., Biegus, J., Pupka, A., et al., 2006. Molecular basics of angiogenesis. Postepy Hig. Med. Dosw., 60:410-415 (in Polish).

Stuttfeld, E., Ballmer-Hofer, K., 2009. Structure and function of VEGF receptors. IUBMB Life, 61(9):915-922. [doi:10. 1002/iub.234]

Swidzińska, E., Naumnik, W., Chyczewska, E., 2006. Angiogenesis and neoangiogenesis - the role in lung cancer and other tumors. Pneumonol. Alergol. Pol., 74(4):414420 (in Polish).

Wada, H., Ura, S., Kitajka, S., et al., 2011. Distinct characteristics of circulating vascular endothelial growth factor-A and C levels in human subjects. PLoS ONE, 6(12):e29351. [doi:10.1371/journal.pone.0029351]

Witmer, A.N., Blaauwgeers, H.G., Weich, H.A., et al., 2002. Altered expression patterns of VEGF receptors in human diabetic retina and in experimental VEGF-induced retinopathy in monkey. Invest. Ophthalmol. Vis. Sci., 43(3): 849-857.

World Health Organization, 2011. Global Status Report on Noncommunicable Diseases 2010. Available from http:// www.who.int $/ \mathrm{nmh} /$ publications/ncd_report_full_en.pdf

Zielonka, T.M., 2004. Angiogenesis. Part II. Factors that modulate the process of formation of new blood vessels. Alergy Asthma Immunol., 9(1):25-31.

\section{中文鳞要:}

\section{本文题目：血糖控制良好的 2 型糖尿病患者血清 VEGF-A 及其受体 VEGFR1 和 VEGFR2 含量检测} A preliminary evaluation of VEGF-A, VEGFR1 and VEGFR2 in patients with well-controlled type 2 diabetes mellitus

研究目的：探讨血糖控制良好的 2 型糖尿病患者血管内皮生长因子-A（VEGF-A）及其受体 VEGFR1 和 VEGFR2 含量及其临床意义。

研究方法: 本文以 31 例血糖得到良好控制且没有明显大血管或微血管病变的 2 型糖尿病患者作为研究对 象 (实验组)，30 位健康志愿者为对照组, 同期检测患者及健康志愿者的空腹血糖、血脂、 糖化血红蛋白水平, 及血清 VEGF-A、VEGFR1 和 VEGFR2 含量, 并加以比较和统计学分析。

重要结论: 研究结果表明, 血糖控制良好的 2 型糖尿病患者血清中的 VEGF-A 及其受体 VEGFR1 和 VEGFR2 的含量和健康志愿者基本一致, 两者无统计学意义, 这可能显示血糖水平的合理控 制能延缓血管并发症的产生。同时， 2 型糖尿病患者的血清 VEGFR2 含量和高密度脂蛋白胆 固醇水平之间存在负相关, 而血清 VEGF-A、VEGFR2 含量和甘油三脂水平之间存在正相关, 这也表明糖尿病患者的血脂紊乱可能参与了血管生成的调节。

关键词组: 2 型糖尿病; 血管生成; 血脂异常; 糖化血红蛋白 